\title{
Einfach besser
}

\author{
Christian Tölken
}

\begin{abstract}
Zwischen 70.850 und 316.100 vollzeitbeschäftigte Pflegekräfte führen Dokumentationen und bedienen Computer. Bürokratie gemessen an der gesamten verfügbaren Arbeitszeit in der Pflege schwankt je nach Untersuchung zwischen 13 und 58 Prozent. Der Zeitaufwand wird mit minimal 16 Minuten und maximal 35 Minuten pro Bewohnerin oder Bewohner und Tag angegeben. Eine Prozessperformance kann helfen, vorhandene Ressourcen besser einzusetzen.
\end{abstract}

Pflege ist Schwerstarbeit. Und deshalb ist es kein Wunder, dass eine ausgebildete Pflegekraft im Durchschnitt nur sechs Jahre zur Verfügung steht. Jede zweite Pflegekraft denkt mehrfach im Jahr daran, den Beruf zu wechseln. Und es sind gerade die unter 40-jährigen Pflegekräfte, die besonders häufig am liebsten weglaufen würden. Dazu kommt: 40 von 100 Pflegekräften leiden an Erkrankungen des Stütz- und Bewegungsapparates. Gerade in Deutschland ist das Image der Pflegeberufe sehr schlecht. Und 63 von 100 befragten Pflegekräften erwarten nicht, dass sich das Image der Pflegeberufe in den nächsten Jahren verbessern werde. Die Pflege verlassen überwiegend die gesundheitlich unbelasteten Mitarbeitenden. Eine ähnlich schlechte Bilanz ist von keiner anderen Berufsgruppe bekannt.

Angesichts der Reform der Pflegeversicherung werden wir wohl mit noch größerem administrativen Aufwand rechnen können, dabei war weniger Bürokratie ein wichtiges Ziel des Gesetzgebers. Schon heute ist absehbar, dass dieses Ziel nicht erreicht wird. Also muss gegengesteuert werden, wenn die Pflege nicht vollständig erstarren soll.

Dabei geht es um das Ziel, die Pflege qualitativ hochwertig und zu- gleich effizient erbringen zu können. Aus Untersuchungen der Tätigkeitsprofile wissen wir: Es steht zu wenig Zeit für die direkte Pflege zur Verfügung.

Die Gründe sind vielfältig. Es gibt derzeit immer noch keine vollständige Übersicht der gesetzlichen Regelungen, die für den Aufbau und Betrieb von Altenheimen relevant sind. Art und Umfang der Gesetze für Alten- und Pflegeheime hängen $u$. a. auch von der Rechtsform der Einrichtung, Tarifgebundenheit und der Gemeinnützigkeit $\mathrm{ab}$. Eine Menge (Rechts-) Holz müssen die Verantwortlichen und alle Mitarbeitenden in den Pflegeeinrichtungen täglich schlagen. Und tatsächlich tummeln sich hier die Gesetzgeber aus Europa, Bund und Ländern. Und nicht genug damit. Auch Vertragsregelungen zwischen Einrichtungen und Kostenträgern haben ihren Anteil am Verwaltungsaufkommen. Über 980 relevante Rechtsvorschriften (Artikel, Absätze, Paragrafen und Unterpunkte) ergeben sich jedoch allein durch eine Auszählung der von Thomas Klie (2003) genannten Normen.

Bürokratie bleibt auch künftig das zweite Gesicht der Pflege und weist auf die Doppelfunktion von Pflege hin: Die Versorgung alter Menschen ist zum einen eine höchst individuelle und persönliche Aufgabe. Sie ist aber auch eine gesellschaftliche Aufgabe, die Aufsicht und Verwaltung benötigt. Bürokratie ist unverzichtbar. Aber sie müsste eigentlich diese Ausmaße nicht annehmen. Will man die Bürokratisierung abbauen, müsste man dort ansetzen, wo sie ihre Wurzeln hat.

Das Grundproblem ist: Es gibt keinen Konsens über die Definition von Qualität in der Pflege. Und es gibt keinen Konsens über das notwendige Tun und deren Finanzierung. Unterschiedliche Akteure (Kostenträger, Verbände, Nutzer, Berufs- und Inter-

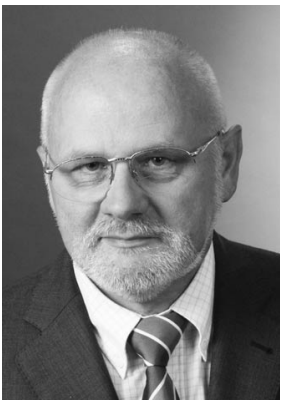

Christian Tölken war von 1981 bis 1982 Vorstandsassistent der Rummelsberger Anstalten, von 1982

bis 1989 Leiter eines Zentrums für Körperbehinderte, von 1990 bis 1993 Personalabteilungsleiter (3.550 Beschäftigte) und von 1994 bis 2004 bevollmächtigtes Vorstandsmitglied der Rummelsberger Anstalten der Inneren Mission und gleichzeitig Leiter der Hauptverwaltung und der Zentralabteilung Finanzwirtschaft, Recht und Grundsatzfragen. Anschließend wurde er bis zu seinem Ausscheiden Ende 2006 der Kaufmännische Hauptgeschäftsführer mit der Verantwortung für 170 Einrichtungen, Dienste, Betriebe, Schulen, Krankenhäuser und rund 6.000 Mitarbeitende. Seit 2007 ist Tölken als Seniorpartner der Consolutions $\mathrm{GmbH} \&$ Co. KG überwiegend von Berlin aus tätig. E-Mail toelken@Consolutions.de

essenverbände) mit teilweise voneinander abweichenden Vorstellungen über die Qualität pflegerischer Leistungen stehen sich im Wege. Qualität hat ja drei Dimensionen. Doch welche ist die Entscheidende? Ist es die Struktur-, die Prozess- oder letztlich doch die Ergebnisqualität? Oder ein Mix von allem? Ein Konsens ist dringend notwendig.

Komplizierte, nicht nachvollziehbare Anforderungen an die Führung einer Pflegedokumentation stellen für Pflegekräfte eine hohe Belastung dar. Es leuchtete ein, dass die Erfüllung nicht transparenter Anforderungen Menschen demotiviert und die Zeit, die für solche Arbeiten aufgewandt wird, besonders lang erscheint. Vereinfachte, gut nachvollziehbare Anforderungen an die Pflegedokumentation, werden nicht als belastend erlebt, wenngleich sie den für die Pflegedokumentation erforderlichen 
Zeitaufwand nicht reduzieren. Im Rahmen des nordrhein-westfälischen Projekts »Referenzmodelle« wurde festgestellt, dass die "gefühlte« Belastung bei der Anwendung vereinfachter Formen der Pflegedokumentation reduziert werden kann, Erwartungen an objektive Zeiteinsparungen jedoch nicht erfüllt werden können.

Im allgemeinen Teil der Begründung der Reform der Pflegeversicherung wird behauptet, die angestrebten Vereinfachungen bei der Pflegedokumentation führten zu Entlastungen bei den Pflegeeinrichtungen und den Pflegekräften im Umfang von wenigstens 80 Millionen Euro. Es wird eine Zeitersparnis von fünf Prozent von der derzeit täglich für 1,15 Millionen Pflegebedürftige aufgewandten Dokumentationszeit von acht Minuten angenommen. Bei einem Lohnansatz von 31,20 Euro pro Arbeitsstunde wird daraus eine Einsparung von circa 86 Millionen Euro errechnet.

Diese Annahme ist nicht gerechtfertigt. Sie steht im Widerspruch zu aktuellen Erkenntnissen aus dem erwähnten Projekt »Referenzmodelle zur Förderung der qualitätsgesicherten Weiterentwicklung 2004-2006« in schem Dienst der Krankenkassen ist nicht vorrangig auf die Gesetzeslage zurückzuführen, sondern auf die mangelnde Kooperation vor Ort. Andere genannte Umsetzungsprobleme, wie beispielsweise die unterschiedlichen Erwartungen von verschiedenen Prüfern, mit denen die Einrichtung konfrontiert werden, lassen eher den Schluss zu, bestimmte Bereiche seien eher zu wenig als zu viel geregelt: Heimleiter machen immer wieder darauf aufmerksam, dass nicht alle Prüfer bei Überprüfungen nach SGB XI die gleichen Erwartungen haben. Deswegen sollte genau definiert werden, welche Listen tatsächlich vorhanden sein müssen und welche Informationen daraus ersichtlich sein müssen (nicht nach dem jeweiligen Wunsch des Prüfers).

\section{Was allgemein geschehen sollte}

Zunächst einmal sollte anerkannt werden, dass Bürokratie eine Folge von rechtsstaatlichen und demokratischen Prinzipien sein kann. Und Bürokratie in der stationären Altenpflege ist als gesellschaftliche Aufgabe notwendig. Nicht zuletzt vor dem Hintergrund der Entstehungsge-
Es ist sinnvoll, die Entbürokratisierungsdebatte stärker im Hinblick auf die eigenverantwortliche Betriebsorganisation der Pflegeeinrichtungen zu führen. Dabei kann man betriebliches Handeln nach folgenden Kriterien prüfen:

- Zweck des Vorgangs oder des Prozesses

- Geeignetheit des Vorgangs oder des Prozesses im Hinblick auf die Erreichung des Zweckes

- Erforderlichkeit des Vorgangs oder des Prozesses (= gibt es gleichwertige Vorgänge oder Prozesse mit geringerem Bürokratieeffekt?)

- Angemessenheit des Vorganges oder des Prozesses (Abwägung zwischen Schutzzweck und bürokratischem Aufwand)

\section{Was in den Pflegeeinrichtungen geschehen kann}

Es gilt, den Erfolg durch Einfachheit zu suchen. Das geschieht durch eine konsequente Prozessoptimierung. Hinter den vielfach beschworenen Wettbewerbsstärken »schlank « und »schnell « steht nichts anderes als der Grundsatz der optimalen Faulheit: weglassen, was überflüssig ist und auf einfache Strukturen und Prozesse konzentrieren.

\section{»Wettbewerbsstärke entsteht durch optimale Faulheit: Weglassen, was überflüssig ist «}

Nordrhein-Westfalen. Angesichts der Realität sind Einsparungen in den Pflegeeinrichtungen aufgrund veränderter Pflichten zur Führung der Pflegedokumentationen nicht zu erwarten.

Weitreichende Veränderungen, beispielsweise hinsichtlich der Finanzierung, Buchhaltung sowie der Qualitätssicherung in der Pflege, haben die Einrichtungen und Träger großen Veränderungen ausgesetzt. Und denen begegnen sie oft genug mit den Antworten der Verwaltung von gestern, gepaart mit den technischen Lösungen von gestern. Die Ressourcen sind einfach knapp. Das Unbehagen an der Bürokratie richte sich nach außen. Dabei wird das interne Verbesserungspotenzial übersehen. In der Praxis richtet sich die Kritik weniger gegen die Inhalte der Gesetze als vielmehr gegen deren Umsetzung im Verwaltungsvollzug. Das häufig benannte Problem der Doppelprüfungen von Heimaufsicht und Medizini- schichte stationärer Altenpflege: Alten- und Pflegeheime stehen in der Tradition von Verwahranstalten, in denen Bewohner lange Zeit so gut wie keine Rechte hatten. Vorschriften wie das Heimgesetz oder das Pflegequalitätssicherungsgesetz gehen auf Regelungsbedarfe ein, die durch Skandale, Presseberichte sowie Prüfungen öffentlich wurden. Drei Ansätze gibt es, um zu einer Verbesserung zu kommen:

- weniger bürokratieaufwendige Vorschriften: Handlungsfeld Gesetzund Verordnungsgebung

- weniger bürokratieaufwendige Ausführung der Vorschriften durch die Verwaltung(en): Handlungsfeld Verwaltungspraxis

- weniger bürokratieaufwendige Umsetzung der Vorschriften in den Betrieben: Handlungsfeld betriebliche Praxis
Vorrang dabei hat zunächst einmal, alle Möglichkeiten zu nutzen, die eine Informationstechnologie (IT) gestützte Pflegeplanung und Pflegedokumentation bieten kann. Und das gilt auch für das Nutzen intelligenter Steuerungs- und Pflegesysteme im Alltag. Genau so wichtig ist, das zu tun, was wirklich gefordert ist. Beispiel: Die MDK-Prüfer fordern immer wieder Listen aller Pflegebedürftigen mit Risiken. Die werden dann auch produziert. Aber fallen wirklich alle Pflegebedürftigen unter diese Anforderung? Nein, nur die, die bei einer Pflegekasse versichert sind. Anderes Beispiel: Häufig wollen die Prüfer Unterlagen zur weiteren Bewertung und Prüfung mitnehmen. Ohne Bedenken werden Unterlagen in beträchtlichem Umfang zusammenkopiert und ausgehändigt. Eine teure freiwillige Leistung der Einrichtungen.

Natürlich braucht Einfachheit Mut. Es ist kein Geheimnis, dass einfache 
Ziele, Strukturen und Prozesse, die klar konzipiert sind und kompromisslos umgesetzt werden, die entscheidenden Merkmale von Spitzenunternehmen sind. Die gibt es auch in der Sozialwirtschaft. Sie haben vielleicht ihren Saint-Exupéry ganz gelesen und nicht nur die Passage vom Schiffsbau und dem Team: «Vollkommenheit entsteht offensichtlich nicht dann, wenn man nichts mehr hinzufügen kann, sondern wenn man nichts mehr weglassen kann. «(In: »Wind, Sand und Sterne $\ll)$

Einfache Lösungen führen zum Erfolg. Pflegeeinrichtungen sind prozessorientierte Organismen, aber so richtig kundenorientiert ist das in der rauen Wirklichkeit nicht immer. Mögliche Stellhebel sind:

1. Den Aufwand und Nutzen in Relation setzen. Das bestehende System ist so angelegt, dass sich Leistungsanbieter am besten stellen, die entsprechend viele Leistungen in dokumentierter Form vorweisen können. Inwieweit die erbrachten Leistungen hilfreich waren, spielt keine Rolle. Papier ist geduldig. Und die heute üblichen Qualitätssicherungsverfahren, Pflegepläne Durchführungsnachweise etc. beweisen letztlich nicht, ob das Angegebene tatsächlich durchgeführt wurde.

2. Das Erkennen ursächlicher Zusammenhänge und das Setzen von Prioritäten fördern. Auf keinen Fall sollten Pflegepläne in einem Wissenschaftsformat verlangt werden. Der ausgewiesene Vorrang von Pflegetheorien und wissenschaftlichen Studien in einzelnen Teilgebieten hat den gesunden Menschenverstand, gepaart mit einer soliden Fachausbildung sowie regelmäßiger Reflexion von Berufs- und Lebenserfahrung fast vollständig in den Hintergrund gedrängt. Indem Checklisten eingesetzt werden, die abwechselnd lediglich verschiedene Teilbereiche beleuchten, wird der ganzheitliche Blick eingeschränkt, der den pflegebedürftigen Menschen in seiner Lebenslage vollständig begreift. So kann es vorkommen, dass um einzelne Details ein Riesenaufwand gemacht wird, der dem Betroffenen zudem noch schadet, weil das Wesentliche durch die Konzentration aufs Detail übersehen wird.

3. Die Verbesserung von Lebensund Erlebenssituation der pflegebedürftigen Menschen muss das Ziel werden. Viele »Qualitätsstandards« stehen in vielen Einrichtungen lediglich parat, um sie im Falle einer Prüfung vorlegen zu können. Was zählt, worauf geachtet wird, ist die korrekte Erfüllung der geforderten Formalitäten. Die Form wird über den Inhalt gestellt.

\section{Was extern passieren muss}

Auch Gesetze und Verordnungen müssen kontinuierlich überwacht und kontrolliert werden, ob die dort enthaltenen Mindestnormen (noch) zeitgemäß sind. Konzepte und die Vorstellungen über Ausstattung (räumlich, personell) und Einrichtungsmerkmale unterliegen im Laufe der Zeit einem Wandel. Das sollte sich auch in Änderungen der Normen wiederfinden. Der moderne demokratische Staat sollte sich der Vorteile der Liberalität und der Autonomie bedienen. Und er muss seinen Bürgerinnen und Bürgern mehr zutrauen.

Erste Empfehlung: Überarbeitung von Heimpersonalverordnung und Heimmindestbauverordnung. Stationäre Einrichtungen werden von einer Vielzahl von Behörden überwacht und geprüft werden. Keine Informationen gibt es darüber, wie häufig eine Einrichtung tatsächlich von den jeweils zuständigen Behörden geprüft wird. Auch kann kaum jemand benennen, welcher Aufwand mit diesen Prüfungen verbunden ist.

Zweite Empfehlung: Quantifizierung des Kontroll- und Prüfaufwands für stationäre Einrichtungen. Immer neue Informationspflichten kommen auf die Pflegeeinrichtungen zu. Wird auf der einen Seite die Leistungs- und Qualitätsvereinbarung aus dem Gesetz gestrichen, taucht sie durch die Hintertür als ein Element der Pflegesatzvereinbarung wieder auf, wenn verpflichtende Leistungs- und Qualitätsmerkmale als verpflichtendes Element dort berücksichtigt werden müssen.

Dritte Empfehlung: Qualität erfordert Bereitstellung der notwendigen personellen und finanziellen Ressourcen. Macht man das nicht, wird man weiter durch die Skandalmedienberichterstattung getrieben werden und Scheindiskussionen führen müssen. Unterdessen werden immer mehr Pflegekräfte mit den Füßen abstimmen dun dem Beruf den Rücken kehren. Wer könnte es ihnen verdenken?
Schon sehr bald wird die Pflege nicht nur viel mehr professionelle Pflegekräfte, sondern auch ein höheres Qualifikationsniveau benötigen. Da eine bedarfsgerechte Bereitstellung von Pflegekräften einen zeitlichen Vorlauf in der Ausbildung erfordert, müssen die Länder, in deren Verantwortung die Altenpflegeausbildung liegt, bereits jetzt mit der kontinuierlichen Erhöhung der Zahl der Ausbildungsplätze beginnen.

Wegen der Veränderungen der Ausdifferenzierung der Bedarfstypen zeichnet sich zudem ab, dass das geschlossene Berufsgruppenkonzept der Pflegeversicherung nur noch begrenzt zukunftsfähig ist. Um den Pflegeberuf zukunftstauglich zu machen und genügend Menschen dafür zu gewinnen, brauchen wir eine auf einem breiten Berufsgruppenansatz basierende Bildungsoffensive für pflegenahe Berufe. Insgesamt muss das Berufsbild der Fachkraft in der Pflege neu strukturiert werden. Eine große Zahl pflegebedürftiger Menschen, insbesondere psychisch Kranke und demente Menschen, benötigen bis zum Erreichen eines fortgeschrittenen Schweregrades ihrer Erkrankung, Behinderung oder Pflegebedürftigkeit weniger medizinisch-pflegerische Leistungen, als vielmehr Assistenz bei der Bewältigung ihres Alltages. Wenn wir hierauf richtig reagieren, kann für viele dieser Menschen ein möglichst langes Verbleiben im bisherigen Wohnumfeld gesichert und stationäre Pflege zumindest hinausgeschoben werden.

Alles zusammengenommen ergibt sich ein großes Veränderungspotenzial. Erschlossen werden kann es über die Fähigkeit, Prozesse zu managen. Das gilt für alle am Pflegeprozess Beteiligten. Sie müssen gemeinsam sicherstellen, die Leistungserstellung mit größtmöglicher Effizienz zu organisieren und gleichzeitig das Leistungsangebot in der vom Markt geforderten Qualität sicherzustellen.

Die Wertschöpfungspotenziale müssen insbesondere von den Pflegeeinrichtungen so gesteuert werden, dass eine optimale Aufteilung dahingehend möglich wird, was in eigener Ressourcenverantwortung und was von Partnern durchgeführt wird. Durch eine nachhaltige Prozessperformance können Umsätze gesteigert und Kosten bei hoher Zufriedenheit der Beschäftigten gesteigert werden. 